ITP-SB-95-06

\title{
Exact ground states of generalized Hubbard models
}

\author{
Jan de Boer* \\ Institute for Theoretical Physics \\ State University of New York at Stony Brook \\ Stony Brook, NY 11794-3840, U.S.A. \\ Andreas Schadschneider ${ }^{\dagger}$ \\ Institut für Theoretische Physik \\ Universität zu Köln \\ Zülpicher Strasse 7ry \\ D-50937 Köln, Germany
}

(March 8, 2022)

\begin{abstract}
We present a simple method for the construction of exact ground states of generalized Hubbard models in arbitrary dimensions. This method is used to derive rigorous criteria for the stability of various ground state types, like the $\eta$-pairing state, or Néel and ferromagnetic states. Although the approach presented here is much simpler than the ones commonly used, it yields better bounds for the region of stability.
\end{abstract}

*email: DEBOER@INSTI.PHYSICS.SUNYSB.EDU

†email: AS@THP.UNI-KOELN.DE 
In recent years there has been an increasing interest in analyzing systems of correlated fermions by constructing the ground state explicitly in certain parameter regimes, but arbitrary dimensions [1]- [8]. In general those ground states have a rather simple structure. Typically one can speak of quasi-classical states where quantum fluctuations are not important and there are no finite-size corrections to the ground state energy. E.g. the fully polarized ferromagnetic state has been in the center of attention. In [7] the corresponding program for superconducting states of the $\eta$-pairing type [9, (7] has been initiated. These $\eta$-pairing states show Off-diagonal-Long-Range-Order (ODLRO) [10] which in turn implies the Meissner effect and flux quantization [10-12], i.e. superconductivity.

The most popular method for the construction of exact ground states was introduced by Brandt and Giesekus [1] and generalized by Strack and Vollhardt [3]. The basic idea is to start with a Hamiltonian $\mathcal{H}_{0}$ with a known ground state $\left|\psi_{N}\right\rangle$ and add operators $\mathcal{P}^{\dagger} \mathcal{P}$ where $\mathcal{P}$ annihilates $\left|\psi_{N}\right\rangle$. Thus $\mathcal{H}_{0}+\mathcal{P}^{\dagger} \mathcal{P}$ also has $\left|\psi_{N}\right\rangle$ as ground state. It is obvious that for a given Hamiltonian $\mathcal{H}$ and ground state $\left|\psi_{N}\right\rangle$ it is usually not easy to find an appropriate $\mathcal{H}_{0}$ and $\mathcal{P}$. Application of this method requires a lot of guesswork and is not very systematic. In addition, one does not gain much insight into the underlying physics by this sort of construction.

Ovchinnikov [5,6] has used a different method to improve some of the results obtained previously. His approach is based on Gerschgorin's Theorem (see e.g. [13]) which gives a lower bound on the ground state energy of the Hamiltonian and thus complements the usual variational principle which gives upper bounds. If those bounds coincide each eigenstate with the corresponding energy is a ground state.

In this paper we apply a much simpler and clearer method for the construction of exact ground states which we call Optimal Ground State Approach (OGS approach). The basic idea is to diagonalize the local interaction and make all local eigenstates which are needed for the construction of the global ground state $\left|\psi_{N}\right\rangle$ local ground states. This usually implies some restrictions on the interaction parameters, typically in the form of inequalities. We will show on a few explicit examples that this method is not only much simpler than the ones described above, but also reproduces or improves the results for all cases considered previously. We like to point out that the OGS method does not only work efficiently for states $\left|\psi_{N}\right\rangle$ which are simple tensor products, but also for more complicated ones, like the $\eta$-pairing states or so-called matrix-product ground states of certain spin-1 chains [14]. A more detailed exposition of the OGS approach and a thorough comparision with the other methods will be presented in a future publication [15].

For any Hamiltonian $\mathcal{H}=\sum_{\langle j l\rangle} h_{j l}$ on a lattice with $L$ sites $(\langle j l\rangle$ denotes neighboring sites) it is usually quite easy to diagonalize the local interaction $h_{j l}$. Suppose now that the lowest eigenvalue of $h_{j l}$ is 0 (e.g. by adding a suitable constant). Clearly 0 is a lower bound for the global ground state energy. The global ground state $\left|\psi_{N}\right\rangle$ is called optimal [16] iff $\mathcal{H}\left|\psi_{N}\right\rangle=0$, i.e. the ground state energy is just the lower bound found by diagonalizing the local interaction.

In the following we will be interested in a generalized Hubbard model with

$$
h_{j l}=-t \sum_{\sigma}\left(c_{j \sigma}^{\dagger} c_{l \sigma}+c_{l \sigma}^{\dagger} c_{j \sigma}\right)+X \sum_{\sigma}\left(c_{j \sigma}^{\dagger} c_{l \sigma}+c_{l \sigma}^{\dagger} c_{j \sigma}\right)\left(n_{j,-\sigma}+n_{l,-\sigma}\right)
$$




$$
\begin{aligned}
& +\frac{U}{Z}\left(\left(n_{j \uparrow}-\frac{1}{2}\right)\left(n_{j \downarrow}-\frac{1}{2}\right)+\left(n_{l \uparrow}-\frac{1}{2}\right)\left(n_{l \downarrow}-\frac{1}{2}\right)\right) \\
& +V\left(n_{j}-1\right)\left(n_{l}-1\right)+Y\left(c_{j \uparrow}^{\dagger} c_{j \downarrow}^{\dagger} c_{l \downarrow} c_{l \uparrow}+c_{l \uparrow}^{\dagger} c_{l \downarrow}^{\dagger} c_{j \downarrow} c_{j \uparrow}\right) \\
& +\frac{J_{x y}}{2}\left(S_{j}^{+} S_{l}^{-}+S_{l}^{+} S_{j}^{-}\right)+J_{z} S_{j}^{z} S_{l}^{z}+\frac{\mu}{Z}\left(n_{j}+n_{l}\right) .
\end{aligned}
$$

Here $c_{j \sigma}$ and $c_{j \sigma}^{\dagger}$ are the canonical Fermi operators, $n_{j \sigma}=c_{j \sigma}^{\dagger} c_{j \sigma}$ and $n_{j}=n_{j \uparrow}+n_{j \downarrow}$ are the corresponding number operators and the $S U(2)$ spin operators are given by $S_{j}^{z}=\frac{1}{2}\left(n_{j \uparrow}-n_{j \downarrow}\right)$, $S_{j}^{-}=c_{j \downarrow}^{\dagger} c_{j \uparrow}$ and $S_{j}^{+}=c_{j \uparrow}^{\dagger} c_{j \downarrow}$.

The first term in (1) is the single-particle hopping, the second one is known as bondcharge interaction. $U$ and $V$ denote the on-site and nearest-neighbor Coulomb interaction, respectively. In addition, we included a $X X Z$-type spin interaction with exchange constants $J_{x y}$ and $J_{z}$ between nearest neighbour sites and a pair-hopping term $Y . \mu$ is the chemical potential and $Z$ the coordination number of the $d$-dimensional lattice.

The local Hamiltonian can easily be diagonalized. Denoting an empty site by 0 , a site occupied by an electron with spin $\sigma=\uparrow, \downarrow$ by $\sigma$, and a doubly occupied site by 2 we find the following 16 eigenstates and their respective energies:

$$
\begin{aligned}
\text { state } & \text { eigenvalue } \\
|00\rangle & E_{1}=\frac{U}{2 Z}+V \\
|\sigma 0\rangle \pm|0 \sigma\rangle & E_{2}^{( \pm)}=\mp t+\frac{\mu}{Z} \\
|\sigma \sigma\rangle & E_{3}=-\frac{U}{2 Z}+\frac{J_{z}}{4}+\frac{2 \mu}{Z} \\
|\uparrow \downarrow\rangle+|\downarrow \uparrow\rangle & E_{4}=-\frac{U}{2 Z}+\frac{J_{x y}}{2}-\frac{J_{z}}{4}+\frac{2 \mu}{Z} \\
|20\rangle-|02\rangle & E_{5}=\frac{U}{2 Z}-V-Y+\frac{2 \mu}{Z} \\
\left|\psi_{ \pm}\right\rangle=\alpha_{ \pm}(|\uparrow \downarrow\rangle-|\downarrow \uparrow\rangle)+(|20\rangle+|02\rangle) & E_{6}^{( \pm)} \\
|\sigma 2\rangle \pm|2 \sigma\rangle & E_{7}^{( \pm)}= \pm(t-2 X)+\frac{3 \mu}{Z} \\
|22\rangle & E_{8}=\frac{U}{2 Z}+V+\frac{4 \mu}{Z}
\end{aligned}
$$

with

$$
E_{6}^{( \pm)}=\frac{1}{2}\left(Y-V-\frac{J_{x y}}{2}-\frac{J_{z}}{4}+\frac{4 \mu}{Z}\right) \pm \beta
$$

and 


$$
\alpha_{ \pm}=\frac{-\frac{U}{Z}+V-Y-\frac{J_{x y}}{2}-\frac{J_{z}}{4} \pm 2 \beta}{4(X-t)}
$$

where $\beta=\sqrt{\left(\frac{V}{2}-\frac{U}{2 Z}-\frac{Y}{2}-\frac{J_{x y}}{4}-\frac{J_{z}}{8}\right)^{2}+4(X-t)^{2}}$.

For the special case $t=X$, which will of some importance in the following, this simplifies to

$$
\begin{aligned}
& \left|\psi_{+}\right\rangle=|\uparrow \downarrow\rangle-|\downarrow \uparrow\rangle, \\
& \left|\psi_{-}\right\rangle=|20\rangle+|02\rangle,
\end{aligned}
$$

with corresponding energies

$$
\begin{aligned}
& E_{6}^{(+)}=-\frac{U}{2 Z}-\frac{J_{x y}}{2}-\frac{J_{z}}{4}+\frac{2 \mu}{Z}, \\
& E_{6}^{(-)}=\frac{U}{2 Z}-V+Y+\frac{2 \mu}{Z} .
\end{aligned}
$$

First of all we look at the $\eta$-pairing states with momentum $P$,

$$
\left|\psi_{N}^{(P)}\right\rangle=\left(\eta_{P}^{\dagger}\right)^{N}|0\rangle, \quad \eta_{p}^{\dagger}=\sum_{j=1}^{L} e^{i P j} c_{j \downarrow}^{\dagger} c_{j \uparrow}^{\dagger} .
$$

As already mentioned these states exhibit ODLRO and are thus superconducting [9].

The $\eta$-pairing state with $P=0$ is an eigenstate of $\mathcal{H}$ for $t=X, 2 V=Y$ [0]]. In order to make it an optimal ground state we first observe that $\left|\psi_{N}^{(0)}\right\rangle$ can be built completely from the local states $|00\rangle,|22\rangle$ and $|20\rangle+|02\rangle$. All three states are already local eigenstates with energy $E_{0}=E_{1}=E_{6}^{(+)}=E_{8}=U / 2 Z+V$, if we choose $\mu=0$. Demanding that $E_{0}$ is the local ground state energy one recovers the result obtained in [7] by using Gerschgorin's theorem:

$$
\begin{aligned}
V & \leq 0, \\
-\frac{U}{Z} & \geq \max \left(2|t|+2 V, V-\frac{J_{z}}{4}, V+\frac{\left|J_{x y}\right|}{2}+\frac{J_{z}}{4}\right) .
\end{aligned}
$$

The state with momentum $\pi$ is an eigenstate of $\mathcal{H}$ for $Y=-2 V .\left|\psi_{N}^{(\pi)}\right\rangle$ is now built from the local states $|00\rangle,|22\rangle$ and $|20\rangle-|02\rangle$. These states have to be made local ground states. Again for $\mu=0$ they all already have the same energy $E_{0}=E_{1}=E_{5}=E_{8}=U / 2 Z+V$. All other energies have to be larger. This yields the following inequalities:

$$
\begin{gathered}
V \leq 0 \\
-\frac{U}{Z} \geq \max \left(2|t|+2 V, V-\frac{J_{z}}{4}, 2|t-2 X|+2 V, V-\frac{J_{x y}}{2}+\frac{J_{z}}{4},\right. \\
\left.V+\frac{J_{x y}}{2}+\frac{J_{z}}{4}-\frac{(t-X)^{2}}{V}\right) .
\end{gathered}
$$


There exist also $\eta$-pairing states with momentum $P \neq 0, \pi$. These appear for instance as ground states of model (11) where $t=X$ and $U \leq-4 t$, but all other interaction constants are zero [7, [17, [18]. These states are eigenstates only for $t=X$ and $Y=V=0$. It is straightforward to derive a similar inequality as for the states with momentum 0 or $\pi$. It is just (8) with $V$ put equal to 0 .

There are other interesting states for which the OGS method can be applied, e.g. the following paramagnetic, Néel, charge-density-wave and ferromagnetic states at half-filling ( $N=L$, where $N=\sum_{j=1}^{L} n_{j}$ is the total number of particles),

$$
\begin{aligned}
\mid \text { para }\rangle & =\prod_{j \in \mathcal{A}} c_{j \uparrow}^{\dagger} \prod_{j \in \mathcal{A}^{\prime}} c_{j \downarrow}^{\dagger}|0\rangle, \\
|N e e l\rangle & =\prod_{j \in \mathcal{B}} c_{j \uparrow}^{\dagger} \prod_{j \in \mathcal{B}^{\prime}} c_{j \downarrow}^{\dagger}|0\rangle, \\
|C D W\rangle & =\prod_{j \in \mathcal{B}} c_{j \uparrow}^{\dagger} c_{j \downarrow}^{\dagger}|0\rangle, \\
|F\rangle & =\prod_{j} c_{j \uparrow}^{\dagger}|0\rangle .
\end{aligned}
$$

$\mathcal{A}$ and $\mathcal{A}^{\prime}$ are arbitrary disjoint sets of lattice points which together span the whole lattice. The states $|N e e l\rangle$ and $|C D W\rangle$ are defined on a bipartite lattice with odd and even sublattices $\mathcal{B}$ and $\mathcal{B}^{\prime}$.

If we want $\mid$ para $\rangle$ to become an optimal ground state we have to make all the states $\left|\sigma \sigma^{\prime}\right\rangle$ with $\sigma, \sigma^{\prime}=\uparrow, \downarrow$ local ground states. From (2) we see that we have to choose $J_{x y}=J_{z}=0$ and $t=X$ in order to get the correct local ground states. These then have the energy $E_{3}=E_{4}=E_{6}^{(+)}=-U / 2 Z+2 \mu / Z$. All other energies have to be higher which finally leads to the condition

$$
\frac{U}{Z} \geq \max \left(2|t|+\frac{2|\mu|}{Z}, V+|Y|,-V+\frac{2|\mu|}{Z}\right) .
$$

For $Y=0$ and $V \geq 0$ this problem has already been investigated in [3]. In this case the bound (14) is better than the one found in [3]. In fact, for $\mu=0$ it is exactly the improved bound found by Ovchinnikov [5] using the Gerschgorin approach.

Similarly, for the Néel state $|N e e l\rangle$ we get the restrictions $t=X$ and $J_{x y}=0$ (from $\left.E_{4}=E_{6}^{(+)}\right)$and the inequalities

$$
\begin{aligned}
& J_{z} \geq 0, \\
& \frac{U}{Z} \geq \max \left(-V-\frac{J_{z}}{4}+\frac{2|\mu|}{Z}, 2|t|-\frac{J_{z}}{2}+\frac{2|\mu|}{Z}, V+|Y|-\frac{J_{z}}{4}\right) .
\end{aligned}
$$

Turning to the state $|C D W\rangle$ we see that this state can be constructed completely from the local states $|20\rangle$ and $|02\rangle$. In order to make these local ground states we have to choose $t=X$, but also $Y=0$. The local ground state energy is then $E_{5}=E_{6}^{(-)}=U / Z-V+2 \mu / Z$. The condition that the remaining energies are higher leads to the inequalities 


$$
\begin{aligned}
V & \geq \frac{|\mu|}{Z}, \\
-\frac{U}{Z} & \geq \max \left(2|t|-2 V+\frac{2|\mu|}{Z},-V-\frac{J_{z}}{4},-V+\frac{\left|J_{x y}\right|}{2}+\frac{J_{z}}{4}\right),
\end{aligned}
$$

which reduces to Ovchinnikov's improved result [5] for $J_{x y}=J_{z}=0$.

The OGS approach shows why a generalization to the non-half-filled case is difficult. Away from half-filling also other states (like $|\sigma 0\rangle \pm|0 \sigma\rangle$ ) would have to be used in the construction of the global ground state. Thus the local ground state degeneracy would have to be larger. In most cases this leads to too much restrictions on the interaction parameters.

For the fully polarized ferromagnetic state (at half-filling) $|F\rangle$ the local ground state is only $|\sigma \sigma\rangle$. Therefore we do not get restrictions like $J_{x y}=J_{z}=0$ or $t=X$ in this case. The local ground state energy is $E_{3}=-U / 2 Z+J_{z} / 4+2 \mu / Z$. This is a lower bound for the other local energies, leading to the inequalities

$$
\begin{aligned}
& -J_{z} \geq\left|J_{x y}\right|, \\
& \frac{U}{Z} \geq \max \left(2|t|+\frac{J_{z}}{2}+\frac{2 \mu}{Z}, 2|t-2 X|+\frac{J_{z}}{2}-\frac{2 \mu}{Z}, \frac{J_{z}}{4}-V+\frac{2|\mu|}{Z}, V+Y+\frac{J_{z}}{4},\right. \\
& \left.V-Y+\frac{J_{z}}{4}-\frac{8(t-X)^{2}}{J_{x y}+J_{z}}\right) .
\end{aligned}
$$

If one is interested in the sector with a fixed particle number $N$, one can regard the bounds as function of $\mu$ and try to find the value of $\mu$ which optimizes these bounds. If a state is a ground state of $\mathcal{H}$ at some fixed particle number $N$, then it is also a ground state of $\mathcal{H}+\mu N$. Hence we only need to require that a state is a ground state for some value of $\mu$, the result for arbitrary $\mu$ follows then immediately. Therefore one can sometimes improve the results of the optimal ground state approach, by first finding inequalities for an arbitrary value of the chemical potential, and subsequently optimize with respect to $\mu$. For example, if we find inequalities $U \geq a+\mu$ and $U \geq b-\mu$, then the best value of $\mu$ is $(b-a) / 2$, and thus the inequality $U \geq(a+b) / 2$.

For (14), (15), and (16) one obviously has to choose $\mu=0$. In the case of the ferromagnetic state the second inequality of (17) can be replaced by

$$
\begin{gathered}
\frac{U}{Z} \geq \max \left(|t|+|t-2 X|+\frac{J_{z}}{2},|t-2 X|-\frac{V}{2}+\frac{3 J_{z}}{8},|t|-\frac{V}{2}+\frac{3 J_{z}}{8},\right. \\
\left.\frac{J_{z}}{4}-V, V+Y+\frac{J_{z}}{4}, V-Y+\frac{J_{z}}{4}-\frac{8(t-X)^{2}}{J_{x y}+J_{z}}\right) .
\end{gathered}
$$

This bound improves the one found in 4 .

We also applied the Gerschgorin approach to the states considered here. In all cases the results are not better than the ones obtained from the OGS approach with optimization with respect to $\mu$ 15.

In summary, we have shown that the OGS approach is - dispite its simplicity - a very useful tool for the construction of exact ground states. It allows to reproduce or even improve bounds for stability regions previously found by different methods and yields better 
insights into the physics. E.g. if the interaction parameters are changed from a situation where the inequalities are satisfied to one where they are not, the ground state typically first is non-degenerate or doubly degenerate (except $|p a r a\rangle$, which is always a highly degenerate ground state), becomes highly degenerate at the OGS bounds (where levels of the Hamiltonian cross), and finally cease to be the exact ground state. In addition, by examining which local eigenstates become the new local ground states one can try to predict some properties of the new ground state once the bounds are violated. We believe that these bounds are as sharp as possible. If an inequality is violated we have a new local ground state which might be used to construct a variational state with an energy lower than that of the "optimal" state. We will come back to these points in a future publication [15] where we also discuss why the OGS approach usually yields bounds which are as least as good as those of the Gerschgorin approach.

We like to thank A. Klümper, V. Korepin, A. Montorsi, E. Müller-Hartmann and J. Zittartz for useful discussions. JdB is supported in part by NSF grant No. PHY-9309888. AS gratefully acknowledges financial support by the Deutsche Forschungsgemeinschaft and through the Sonderforschungsbereich 341. He also thanks the Institute for Theoretical Physics in Stony Brook, where this work was started, for its hospitality. 


\section{REFERENCES}

[1] U. Brandt, A. Giesekus, Phys. Rev. Lett. 68 (1992) 2648

[2] R. Strack, Phys. Rev. Lett. 70 (1993) 833

[3] R. Strack, D. Vollhardt, Phys. Rev. Lett. 70 (1993) 2637

[4] R. Strack, D. Vollhardt, Phys. Rev. Lett. 72 (1994) 3425

[5] A.A. Ovchinnikov, Mod. Phys. Lett. B7 (1993) 1397

[6] A.A. Ovchinnikov, J. Phys. CM6 (1994) 11057

[7] J. de Boer, V.E. Korepin, A. Schadschneider, Phys. Rev. Lett. 74 (1995) 789

[8] A. Montorsi, D.K. Campbell, preprint cond-mat/9502027

[9] C.N. Yang, Phys. Rev. Lett. 63 (1989) 2144;

C.N. Yang and S. Zhang, Mod. Phys. Lett. B4 (1990) 759

[10] C.N. Yang, Rev. Mod. Phys. 34 (1962) 694

[11] G.L. Sewell, J. Stat. Phys. 61 (1990) 415

[12] H.T. Nieh, G. Su, B.M. Zhao, Phys. Rev. B51 (1995) 3760

[13] R. Zurmühl, Matrizen (Springer, Berlin, 1964)

[14] A. Klümper, A. Schadschneider, J. Zittartz, Europhys. Lett. 24 (1993) 293

[15] J. de Boer, A. Schadschneider, in preparation

[16] J. Zittartz, private communication

[17] A.A. Aligia, L. Arrachea, Phys. Rev. Lett. 73, 2240 (1994)

[18] A. Schadschneider, Phys. Rev. B51 (in press) 